\title{
Preface Issue 4-2012
}

\author{
Hans-Christoph Grunau
}

(C) Deutsche Mathematiker-Vereinigung and Springer-Verlag Berlin Heidelberg 2012

"Foundations of Discrete Optimization: in transition from linear to non-linear models and methods." This is the title of the survey article by Jesús A. De Loera, Raymond Hemmecke, and Matthias Köppe in which the authors summarise a number of recent developments in discrete optimisation. These range from improving, extending, and discussing classical algorithms from linear optimisation to nonlinear transportation problems. A common feature of most real life optimisation problems is the huge number of variables and hence, the need for efficient, i.e. fast, algorithms. For a long time there has been an intimate relation between optimisation and convex geometry and in this respect it would be particularly interesting to find good bounds for the so called diameter of polytopes. The related prominent Hirsch conjecture was discussed in Issue 2-2010 of the Jahresbericht by Edward Kim and Francisco Santos. Although this particular conjecture was disproved by the latter author while their survey article was in press, the struggle to find good diameter bounds remains. Jesús De Loera, Raymond Hemmecke, and Matthias Köppe explain further how a more refined modelling changes linear problems into nonlinear ones and how algebraic, geometric, and topological techniques enter discrete optimisation in order to tackle the new challenges. For more detailed information and proofs the authors refer to their corresponding recent monograph.

Anybody, irrespective of the individual fields of interest, who has ever visited the Oberwolfach institute will know the name of Horst Tietz. The reason is that a former student of his established a trust named after his advisor to support the institute. Georg Schumacher reviews not only the mathematical and academic achievements of Horst Tietz, who died on 28th January 2012, but also focusses on some details

\footnotetext{
H.-Ch. Grunau ( $₫)$

Institut für Analysis und Numerik, Fakultät für Mathematik, Otto-von-Guericke-Universität, Postfach 4120, 39016 Magdeburg, Germany

e-mail: hans-christoph.grunau@ovgu.de
} 
from his life which are really worth thinking about. Due to his Jewish roots Horst Tietz was deported in 1943 first to the concentration camp Breitenau and later to Buchenwald. It is somehow a miracle that Horst Tietz survived the horrible years of this most degrading captivity. In spite of these traumatic experiences he stayed in Germany, finished his studies, and started an academic career just after the end of the Nazi-regime.

Recently released books on $p$-adic Lie groups, Feynman motives, and modern classical homotopy theory are extensively discussed and reviewed.

Hans-Christoph Grunau 